Servicio de Tisiología

"Hospital Arriiarán".

Sanatoric "Los Guindas"

del Consejo de Detensa del Niño.

\title{
LA ATElectasia pUlmonar EN El NINO Y SUS RELACIONES CON LA TUPERCULOSIS PRIMARIA
}

Pot los Dress. JORGE PEÑA CERECEDA. ERNESTINA PEÑA D.

y LUCILA CAPDEVILLE.

\section{Introducción.}

Este trabajo se refiete al estudio de la atelectasia en el niño, principalmene en relación con la tuberculosis primaria.

Hemce estudiado de preferencia los distintos métodos que se han propuesto para el diagnóstico de esta modalidad clínica de la tuberculosis infantil. especialmente los que se refieren a la broncoscepía y a la radiología, por considerarlos los más eficaces y los más fáci'es de realizar en la práctica.

Además hemos podido realizar el examen anátomo-patológico de seis casos de atelectasias en diversos periodos de su fvolución, cbeervados clínica y radiológicamente durante un lapso más o menos largo.

La exposición la hemos crdenado de la siguiente manera:

I. Generalidades y licetatura sobre la materia.

II. Estudio estadístico sobre la frecuencia y localizaciones de la atelectasia en nuestro medio.

III. Definición, clasificación $y$ patogenia de la atelectasia.

IV. - Estudio anátomo-patológico macro y microscópico de seis observaciones de atelectasia. 
V. - Sintomatologia clínica.

VI. - Relaciones de la atelectasia con las enfermedades infecto-contagiosas.

VII. -- Métedos de diagnóstico:
a) Radiológico:
b) Broncoscópico;
c) Manométrico:
d) Pleuroscópico;
e) Neumotórax diagnóstico.

VIII. - Diagnóstico diferencial.

IX: - Conclusiones.

X. - Bibiiografía.

I. -- Generalidades.

Se acostumbra dividir la tubercu'osis infantil en dos grupos: taberculesis de primo-infección $\mathrm{y}$ de re-infección. Aunque esta clasificación tiene ciertc valor clínico en muchos casos, sin embargo, no es posib!e encuadrar ciertas formas intermediarias en uno $\mathfrak{a}$ otro grupo.

Para facilitar nuestro eștudio, consideraremos como țuberculosis de primc-infección a todas aquellas formas que tengan alguna relación con la tuberculosis de los ganglios tráqueo-brónquicos, que son los que determinan el carácter de las lesiones puimonares. directa o indirectamente. Incluiremos, por consiguiente, en este grupo a las llamadas infiltra. ciones primarias, post-primarias, secundarias, etc., que no tienen una individualidad bien definida y que corresponden más bien a distintas modalidades de un mismo proceso en relación con la tuberculosis de los ganglios mediastínicos o con alterationes inflamatorias de la mucosa bronquial.

Como tuberculosis de reinfección consideraremos a todas aquellas formas que se desarroilan a nivel de antiguas lesio. nes del periodo primario, principalmente nódulos hematogenos pulmonares. Este aspecto ha sido estudiado extensamente en un trabajo de Peña Cereceda y Feldman C. (1), por lo que no nos referiremos a este aspecto de la tuberculosis in. fantil.

En los últimos años se ha hecho una revisión considerable de los conceptos clásicos de la tuberculosis primaria en ei niño, a partir de los trabajos de Eliasberg y Neuland, de la clínica de Czerny (2), en 1920. Estos autores describieron. 
en lactantes $y$ en niños mayores, formas crónicas que no tenian el aspecto clínico al radiológico de la tuberculosis pui. monar: pero que aparecía en niños tuberculizados y con lis siones tuberculosas ganglionares o de otros órganos.

Este proceso pulmonar se caracteriza por sombras lob?res, o de mayor extensión. de carácter represivo, homogéneas. de límites más o menos precisos que, al examen clínico. sólo dan manifestaciones mínimas idisminución de la sonoridad. tespiración ruda, a veces estertores); en otras ccasiones no presentan ninguna sintomatología.

- Los autores llaman la atención hacia e? hecbr de aue estas lesiones no guardan relación con la sintomatología general. aue sólo se manifiesta por febriculas detención de in curva de pesc. palidez. etc., y 10 que es más interesante. 1? Evolución es siempie favorable en lo que se tefiere a estas sombras pulmonares. En cuanto a las favorables, se deben más bien a lesiones tuberculosas concomitantes.

Eliesberg y Neuland denominan a este tipo de condenración. "infiltración epituberculosa". Esta denominación es bastante ambigua, porque abarca gran número de lesiones Fulmonares de los niños, que tienen caracteres más o menos semejantes; lo único que permite diferenciarla es su curso favcrable. después de una evolución más o menos larga. F! diagnóstico de epituberculosis no puede hacerse con soguridad. rinc despues de una observación prolongada qus permita comprobar su regresión.

A partir de estos trabajos se ha multiplicado a terminologia según las investigaciones o los conceptos de cada autor. Para Söderling (3), los términos de "infíltración perifocal de Redeker". "infiltraciones primarias". "esplenoneumonía de Grancher". "infiltración colateral de Tendeloo". "condensación tulmonar tuberculosa crónica curable". "infiltración tutarculosa beniena", atelectasia en la tuberculosis de los ganalios hiliares de De Bruin'. "paratuberculosis de Engel", "inflamación gelatinosa de Laennec". "neumonía :iescamativa de Buhl". "protein-neumonía de Cornet", etc. son todos sinónimos.

Simon y Redeker (4) crean el término de "infiltración Ferifocal". con el cual designan las sombras que se desarrollan en las vecindades de un foco tuberculoso y consideran a estas lesiones, que no tienen el aspecto de tejido tuberculoso, como producidas por endotoxinas y no por el bacílo mismo.

Hutzler-Oppenbeim (5), sostienen una opinión semejante y la basan en estudios experimentales en animales. 
Tendeloo y Ranke i6) creen qué la inflamación perifocal también es debida a la endotoxina. Este último autor le atribuye gran importancia a la acción de la toxina de los bacilos circulantes en el período secundario.

Prosoroff (7) en 1929, fué è primero que identificó : la epituberculosis como una atelectasia. Comparó las lesiones pulmonares que se producían por la compresión de los bronquios por los tumores intratorácicos. con las provocadas por los ganglios tuberculosos. Propone la denominación de "espleno-neumonia cbstructiva atelectásica de la tuberculosis de los ganglios biliares".

Roessle, en 1935 (8), hace una tevisión muy completa de los trabajos de Ghon. Epstein, Eliasberg y Neuland, sobre las infiltraciones inespecíficas. Este autor tiene el mérito de taber sido el primero en hacer notar la importancia que ticnen los infartos de los ganglios mediastínicos en la forma. ción de las combras benignas pulmonares, que èl cataloga como atéectasias. Roessle, sobre una base de 11 casos escu. diados desde el punto de vista clínico y anátomo-patológic.? $y$ que hatian sido catalogados como epituberculcsis. demuestra que estas lesicnes correspondian a atelectasias. Divide su material en dos grandes grupos: 1." los llamados "casos limtios", o sea, aquéllos en los cuales la sombra apareció en forma progresiva y que sanaron anatómicamente en forma completa. Estas atelectasias se debieron a compresión de los bronquios por gangios hipertrofiados y sin lesiones específicas de su pared o de su lumen. Para Roessle se trataba de atelectasias sin infección especifica que regresaron junto con los ganglios determinantes de la compresión. "En el segundo gru. po de cascs. que Roessle denomina "no limpios". la atelecta. sia sería determinada por ia perforación de un ganglio en un bronquio. con diseminación tuberculosa de la zona atelectasiada. En estos casos la rizgresión espontánea no seria posible.

Aunque los concepros de Roessle ban sido criticados por muchos autores. en material de observación aportado por él al estudio de las atelectasias. constituye hoy día la base más sólida para el conocimiento de este tipo de lesiones en la infancia.

Para De Bruin. en 1936 (9), muchos casos de epituber. culosis son atelectasias consecutivas a estenosis del bronquio principal. Subraya las dificultades que existen para hacer el diagnóstico de la atelectasia por los rayos $X$. cuando éstas se producen gradualmente o cuando hay adherencias pleurales que impiden las dexviaciones del mediastino. 
Soederling (3), en 1939, hace un resumen muy completo de la literatura sobre estas lesiones del niño y llega a la conclusión de que el cuadro clínico y radiológico de la epitutuberculosis, se debería a la complicación de una tuberculosis puimonar exudativa por un colapso lobar. Propone el término de "colapso" en lugar de atelectasia. El autor no aporta observaciones anátomo-patológicas propias sobre el particular y su casuística se limita solamente a algunas observacioneg clínico-radiológicas.

Entre nosotros. las primeras publicaciones sobre atelectasias pulmonares determinadas por ganglios tuberculoses tráquec-brónquicos, fueran hechas por Scroggie y Romero (10). en una comunicación al II Congreso Nacional' de Pediatría en 1937.

L'odrá Banzá (11), de la Clínica Pediátrica Universitaria del Prof. A. Scroggie, publica un estudio muy completo scbre atelectasias en la infancia.

Este trabajo se refiere especialmente al estudio broncográfico de las obstrucciones del árbol bronquial en niños con manifestaciones clínicas y radiológicas de atelectasias.

K. Terolan en 1940. (12). llama la atención sobre Ins leciones twherculosas de los brongurins por vaciamiento, en su lumen. de focos primarios caseificarlos.

Cita observaciones de atelectasias oroducidas por la $o c^{-1}$ sión de un bronquio distal por exudados provenientes de los oulmones vecinos. o por nequeños tubérculos productivos en los al+pacderes de las paredes del bronauio.

En algunos años el proceso tuberculoso bronauial h? curado completamente, de jando una cicatriz obstructiva en un hronquio de , pequej̃o calibre, que determina una atelertasia. En otras ocasiones, el proceso se debería a la compresión del $\mathrm{pu}^{\prime \prime}$ món por un foco primario caseificado, dando lugar a uṇa atelectasia en el área periférica, vecina a dicho foco; pero. ia mayoría de las veces, la lesión se deberia, según este autor, a procesos 'de tuberculosis endcbronquiales, caseosos o fibrinosos, que producirían un estrechamiento del lumen de los bronquios.

La casuística de Terplan es de 5 casos, estudiados desde el punto de vista anátomo-patológico. Volveremos a hablar sobre sus investigaciones en el capitulo correspondiente a la anatomía patológica.

Edna Jones, T. N. Rafferty y H. S, Wi'lis en 1940 (13). estudian varios casos referentes a epituberculosis, que identifican como atelectasias producidas por obstrucción bronquial. 
Igualmente E. M. Kent. en 1940 (14), llegan a conclusiones semejantes a la de los autores más arriba mencionados.

Por último, J. Menegbello y C. Sniths (15), en un tratajo reciente, bacen notar la frecuencia con que se presenta el enfisema junto a las lesiones atelectásicas en el curso de inflamaciones endcbronquiales diversas, determinadas por gangìies tuberculosos tráquieo-brónquicos.

\section{Frecuencia y localizaciones de la atelectasia.}

Nuestro estudio está basado en la revisión de 3,418 observaciones recogidas en el Servicio de Tuberculosis del Hospital "M. Arriarán", desde el año 1937 hasta 1942 y en el Servicio de Tuberculosis del Consejo de Defensa del Niño, desde 1934 hasta 1942.

La proporción de atelectasias, comprobadas clínica y radiclógicamente $y$ en algunos casos por la autopsia alcanza a 249. $(7,78 \%)$.

No hemos considerado en este grupo, aquel'as atelectasias cuyo diagnóstico no ha sido bien precisado, ya sea por falta de datos radiográficos o por insuficiencia de observación: nos hemos limitado solamente a escoger 35 observaciones controladas durante un tiempo superior a 6 meses (muchas de ellas se han pcdido observar durante 2 y 5 años), tanto desde el punto de vista clínico-tadiológico, como broncoscópico, pleuroscópico, etc.

Se ha llegado a practicar la autopsia en 6 casos fallecidos por una enfermedad intercurrente o por una forma aguda de tuberculosis y en ellos se ha logrado practicar los exámenes macro y microscópicos de las lesiones.

datos.

El estudic detallado de las atelectasias da los siguientes

En cuanto a su extensión:

\begin{tabular}{|c|c|c|c|}
\hline & $\mathrm{N} .{ }^{\circ}$ de caso. & Detechos & Izquierdos \\
\hline Atelectsisias totales .. . & $8(22.86 \%)$ & $4(50 \%)$ & $4(50 \%)$ \\
\hline & ' & & \\
\hline Atelectasias parciales & $27(77,14 \%)$ & $21(77.77 \%)$ & $6(22,22 \%)$ \\
\hline
\end{tabular}

Las formas parciales pueder dividirse según su localización: 
TOTAN DE CASOS: 27

\begin{tabular}{|c|c|c|c|c|c|}
\hline \multicolumn{2}{|c|}{ Tercio superior } & \multicolumn{2}{|c|}{ Tercio midio } & \multicolumn{2}{|c|}{ Tercic inferior } \\
\hline \multicolumn{2}{|c|}{$16(59,25 \%)$} & \multicolumn{2}{|c|}{$5(18.52 \%)$} & \multicolumn{2}{|c|}{$6(22,22 \%)$} \\
\hline Der. & Izq⿴. & Der. & Izq. & Der. & Izq. \\
\hline 12 & 4 & 4 & 1 & 5 & 1 \\
\hline
\end{tabular}

De las cifras expuestas se desprende que la gran mayoría de las atelectasias se localizan en el lado derecho $y$ de praferencia en el tercio superior, lo que concuerda con las estadisticas de la mayoría de lcs autores. Siguen, en orden de frecuencia, las del tercio inferior y medio; sin embargo. estos porcentajes no corresponden exactamente a la realidad, porque no siempre es posible precisar la verdadera localización de las lesiones en los lóbulcs correspondientes, va qư las imágenes radiológicas se superponen, especialmente en las regiones yuxta-cisurales. La radiografía lateral permite, con frecuencia, diferenciar las lesiones de los lóbulos medios e inferiores; pero, en lo que respecta al lóbulo superior. sobre todo cuando está afectado en su parte baja, el diagnóstico de localización resulta extremadamente dificil.

Suele suceder tambièn que se produzca al mismo tienpo una atelectasia de la parte inferior del lóbulo superior y de la región más alta del inferior, lo que dificulta aun más el diagnóstico.

Respecto a la evolución de las atelectasias no podemos dar datos exactos, porque no nos ha sido posible establecer el diagnóstico al comienzo de la enfermedad, síno en un número muy reducido de ellas. En la mayoría de nuestros casos, Ia atelectasia se borró en un plazo aproximado de 5 meses, tomando en consideración sclamente la fase aguda, o sea. desde la iniciación del proceso hasta la formación de lesiones induradas de diverso orden.

Las edades de nuestros niños cscilan entre 2 meses y 10 años, periodos de la vida en que predominan las formas de primo-infección; sin embargo. la gran mayoría de las atelectasias corresponden a lactantes que dan las formas de mayor extensión y de aspecto más característico. En los niños maycres, especialmente entre los escolares, sólo se ven formas localizadas, sin sintomas generales apreciables y que corresponden a atelectasias producidas por estrechamientos fibrosos de los bronquios vecinos a ganglios en cicatrización. 
Debemos hacer notar que todos los casos que hemos otservado, siguietón una evolución benigna "quad vitam", pero la gran mayoría de ellos dejaron procesos residuales como bronquiectasias u otras secuelas de carácter definitivo.

\section{Definición, clasificación y patogenia de la atelectasia.}

Difinición. - (Del griego: ateles: incompleto y ektasis: extensión).

Si nos atenemos a las definiciones clásicas de Chadourne. Sergent y Durant que entienden por atelectasia un pulmón privado de aire en forma absoluta, aumentado de consistencia, reducido de volumen, de coloración rojo-oscura, congettionado, con exudado intraalveolar edematoso o hemorrágico. con transformación del epitelio alveolar plano en epi. téio cúbico, como en el estado fetal, que se produzca solamente en tejidc sano y que sea reversible, nos encontramos con un cuadro anatómico muy difícil de comprobar en la clínica, salvo en la forma fetal, o en aquellos casos de obstrucciones bronquiales totales (cuerpos extraños) y solamente en su fase aguda.

Este concepto se ha ampliado en los últimos años y la mayoría de los autores, especialmente alemanes y norteamerícanos, lo hacen extensivo también a las atelectasias por comtresión y a las formas crónicas, de tal manera, que podría definirce por atelectasia un pulmón desprovisto de aire, reducido dz vclumen y funcienamiento excluídz de la respitasión: cin estar forzos mente congestionad: o edimatizado cn ful: tcavidades alveolares.

Además, se considera que la reversibilidad no es una condición absoluta para el diagnóstico de atelectasia, ya que tste ferómeno no se observa en las formas crónicas que están fuertemente induradas. Alguncs autores entienden por atelectasia una disminución de la función tespiratoria, concepto que se confunde con el de colapso. Se habla da atelectasia en los casos en que falta el aire en los alvéolos, en que existe una inhibición de la función respitatoria y una congestión con edema intra-alveolar; mientras que en el colapso, persiste la función respiratoria, aunque en un grado muy disminuído y los vasos sanguíneos disminuyen su calibre (isqué mia for compresión). Por último, en el colapso hay disminución de la presión negativa endotorácica, la que está aumentada en 'a atelectasia. Además, la zona colapsada es transparente a los rayos $X$, pues contiene aire: mientras que ten la atéectasiada hay opacidad. 
Clasificación. - Existen numerosas clasificaciones de las atelectasias: pero casi todas tienden a englobarlas en dos grandes grupos:

a) Endógenas o por obstrucción bronquial, y b) Exógenas o por compresión. Todavia podríamos diferenciar las formas lobares o masivas y las parciales, que comprenden la atelectasia de los lobulillos, las perifocales y las pericavitarias. Por último, siguiendo a Sayé (16), podemos dividirlas en agudas y crónicas.

Las atelectasias por causa endógena se deben a obstruc. ciones de los bronquios por exudados caseosos o muçsos, sangre (atelectasias post-hemoptoicas). cuerpos extraños, vegetaciones benignas o malignas de la mucosa bronquia!, anomalías, inflamaciones o granulaciones de la mucosa, tamcres de la pared o de la mucosa (quistes), etc. Estas causas preducen la interrupción total o parcial del aire exterior hacia los alvéolos. En los niños las atelectasias se deben principalmente a inflamaciones o ulceraciones de la mucosa bronquial en el sitio correspondiente a ganglios' tuberculosos. En otras ocasiones, estas obstrucciones se deben a anillos fibrosos que estrechan el lumen y producen un punto de menor resistencia en una zona determinada de la pared bronquial.

Las atelectasias por causa exógena no constituyen un cuadro clínico bien diferenciado. Se habla de atelectasias por compresión en los casos en que existe una abolición de la función respiratoria, con desaparición del aire alveolar y flegamiento de las paredes de los mismos.

Las características anátomo-patológicas de las atelectssias las estudiaremos en el capítulo correspondiente.

Patogenia. - Estudiaremos principalmente las atelectasias por causa endógena o por obstrucción bronquial, que son las que hemos encontrado casi exclusivamente en el niño. Según Chevalier Jackson y Chevalier L. Jackson (17), el tórax puede considerarse como una cavidad dividida en dos partes por un tabique (mediastino) y que es hermética al aire. Cada una de estas cavidades está ocupada por una bolsa de aire representada por. los pulmones. Cuando el aire penetra en los alvéolos, éstos se distienden en conjunto: porque actúa sobre ellos, uniformemente, la presión atmosférica. Si se obstruye la entrada dẹt aíre en un lado, el pulmón disminuye de volumen y el mediastino se desvía hacia el lado del pulmón afectado. 
Los autores citados han realizado observaciones muy importantes por medio de la broncoscopía y que resumiremos brevemente:

La obstrucción de un bronquio puede ser total o parcial y sus causas más frecuentes son: exudados, secreciones viscosas, sangre, cuerpos extrañcs, etc. Cuando la obstrucción bronquial es total, se produce la atelectasia en el territcrio pulmonar correspondiente.

Si ia obstrucción es incompleta se observa el estrechamiento del lumen bronquial y el aire produce al pasar, un estriclot o jadeo caracteristico, tanto en la inspiración conıo en lis espiración.

Las obstrucciones parciales pueden producirse también for an mecanismo valvular, del cual distinguimos dos tipos:

a) Si el lumen bronquial está permeable solamente dura.nte la inspiración y se cierra durante la espiración. se obtiene enfisema.

b) A la inversa, cuando el bronquio es permeable solamente durante la espiración y se cierra en la inspiració:n, se produce la atelectasia. Los tipos descritos pueden alternarse de tal modo, que un enfisema se transforma rápidamento en una atelectasia por inversión del mecanismo de válvula.

Para comprender mejor la producción de ruidos anormalcs en los bronquios o las alteraciones de los signos clínicos pulmonares, es preciso tener presente los siguientes hechos:

1) El aire que pasa por un bronquio normal no produce ningún signo físico.

2) Los ruidos anormales se producen por las siguientes causas:

a) Alargamiento o estrechamiento del lumen del bronquio; b) Tumefacción de la mucosa: c) Proyección de la pared brcnquial hacia el interior; d) Cuerpos extraños: e) Secreciones.

3) La atelectasia y el enfisema pueden coexistir en tal forma, que en una rama bronquial exista una atelectasia y en la otra enfisema. Esto nos demuestra que los enfisemos no son compensatorios como se los ha creido hasta hace Foco, sino que su mecanismo es el mismo que el de la atelectasia (Véase Observaciones N.os 12 y 25).

Debemos hacer una mención especial de las atelectasias amyzicas, descritas por el Profesor Westenhoefer en 1919 (18) y estudiadas posteriormente en la tesis de $\mathrm{H}$. Rodríguez (19), en la que analiza 62 casos de lesiones de este tipo. 
La atelectasia amyzica (del griego: falta de succión), se produciría por la falta de acción de las paredes torácicas sobre el pulmón. lo que permite que una porción de este órgano permanezca inmovilizado durante un tiempo más o menos largo.

Las condiciones para la producción de estas atelectasias serian las siguientes:

1) Que exista un obstáculo (aire. líquido, tumores, (te.) que separe el pulmón de la pared torácica e impida. por consiguiente. que ésta distienda el pulmón por medio de los movimientos activos.

2) Falta da actividad del tórax en niños caquécticos 0 debilitados, que hayan permanecido mucho tiempo en cama.

3) Elevación o inmovilización del diafragma por gases, tumores, aneurismas, etc., siempre que actúen en forma permanente.

4) En el tórax raquitico dę los niños o en reblande. cimientos extensos de la pared torácíca.

5) En las toracosplastias extrapleurales, xifoescoliosis, tumores del mediastino, ete.

La condición principai para que se produzca una ate. lectasia amyzica. es que exista una disminución de la presión negativa endopléural, que permita al pulmón retraerse, pero. sin que haya una verdadera compresión del órgano. (Véase ia Otservación N. ${ }^{\circ}$ ).

Mencionaremos también las atelectasias por compresión que se producen por neumotórax, tumores, aneurismas, aumentos de volumen del corazón. exudados a transudados en ta pleura, etc. Estas diferentes causas obran sobre la superficie pulmonar, impidiendo en primer lugar, entrar el aire en los alvéolos y expulsando después activamente el aire de reserva y el residual. También se acepta por algunos autores el mecanismo de la reabsorción del aire por la sangre.

De esta manera se obtienen atelectasias parciales o totales, en las cuales el órgano aparede disminuído de tamaño. aumentado de consistencia, de color gris café, más o menos seco al corte $y$ sin aire.

Al examen microscópico aparecen los vasos sanguíneos plegades y sin sangre, es decir, que se trata de una atelectasia anémica (atelectasia pálida de Letulle), mientras que en la atelectasia amyzica el color es rojo. 